\title{
Development of Inchworm Type Pipe Inspection Robot using Extension Type Flexible Pneumatic Actuators
}

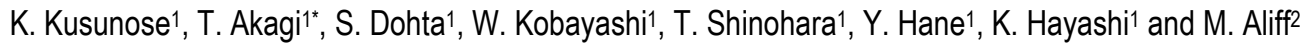 \\ ${ }^{1}$ Okayama university of science, 1-1, Ridai-cho, Kita-ku, Okayama, 700-0005, Japan \\ Phone: +81-86-256-9786; Fax: +81-86-255-3611 \\ ${ }^{2}$ Malaysian Institute of Industrial Technology, Universiti Kuala Lumpur, Persiaran Sinaran IImu, Bandar Seri Alam, 81750 Johor, Malaysia
}

\begin{abstract}
In the case of damp and wet pipes, pipe inspection robots using pneumatic actuators offer advantages such as no electrical leakage and short circuit. In the previous study, a robot consisting of sliding/bending mechanisms using parallel arranged three extension type flexible pneumatic actuators and two holding mechanisms was successfully developed. In order to use the robot in thinner pipe, a novel and simpler propulsion mechanism utilising the difference of frictional force moving forward and backward are proposed and tested in this work. There are two mechanisms, which are "wriggling type" and "cilia type". The "wriggling type" mechanism moves forward by wriggling its body while the "cilia type" mechanism moves by using plate type cilia that covered on the mechanism. Both mechanisms have been tested in the pipeline. As a result, it can be confirmed that the cilia type propulsion mechanism can travel in the pipe with accumulated water. It can be found that the mechanism can easily travel through corners while twisting its body by giving bending motion toward any direction.
\end{abstract}

ARTICLE HISTORY

Revised: 3 rd July 2020

Accepted: $19^{\text {th }}$ July 2020

\section{KEYWORDS}

Extension type flexible pneumatic actuator, Pipe inspection robot, Sliding/bending mechanism, Wriggling type propulsion mechanism, Cilia type propulsion mechanism

\section{INTRODUCTION}

In 2014 , about $12.1 \%(660,000 \mathrm{~km})$ from the total length of the Japan water supply pipeline exceeded the statutory life of 40 years [1]. As only $0.76 \%$ of the total pipe is changed every year, it seems to take 130 years to renew the whole pipeline here. In addition, water pipelines are complicated and consist of numerous corners and joints. If an excavation works is selected to inspect the entire pipes, it is costly and time-consuming. Therefore, various inspection methods such as a fibre scope, dowsing and a wheel type inspection robot have been developed. However, a manipulated fibre scope has a disadvantage that the searching area is inadequate because the pushing force is not sufficiently transmitted. Consequently, pipe inspection robots that can travel by themselves have been developed by various companies and researchers [2-4]. The cost of pipe inspection can also be reduced by using these robots. Hence, inspection robots need high mobility in a pipe. In ideal, it is more desirable that the shape of the robot body changes naturally because of the low energy consumption and reducing the time for travelling. In addition, a fluidic actuator is practical and safe to be used in damp and wet conditions such as in water supply pipe because the actuator does not use electricity as their primary energy and has no risk of electrical leakage and short circuit [5-8]. Based on this concept, a piping inspection robot that consists of a sliding propulsion mechanism and a holding mechanism using extension type flexible pneumatic actuators (EFPAs) has been proposed and tested in our previous study $[9,10]$. The robot could travel smoothly in the pipe with an inner diameter of $100 \mathrm{~mm}$ [10]. Unfortunately, it is difficult to apply to thinner pipes due to the complex mechanism that includes sliding propulsion and pipe holding mechanisms. Thus, it is necessary to develop a simpler propulsion mechanism. The purpose of our study is to develop a simpler propulsion mechanism that can travel in a thinner and complex pipe with an inner diameter of less than $100 \mathrm{~mm}$.

In this study, two types of propulsion mechanisms that do not use the holding mechanism for simplification are proposed and tested. One is wriggling type mechanism that can move forward by wriggling its body using EFPAs. Another is cilia type mechanism that can move by using some plate type cilia covered on the mechanism. Both mechanisms can travel in the pipe by utilising the difference of frictional force moving forward and backward. In this paper, the construction and operating principle of both mechanisms are described. Also, the mobility using both mechanisms were through the driving test of these mechanisms in narrow straight and bending pipes.

\section{Previous Pipe Inspection Robot}

Figure 1 shows the view of the whole pipe inspection robot developed in our previous study [10]. The robot consists of a sliding/bending mechanism and two holding mechanisms using EFPAs. Two holding mechanisms are placed at the ends of the sliding/bending mechanism. The coil type tube is used to supply air from the end of the robot to the top end of the holding mechanism. The total length and mass of the robot are about $0.3 \mathrm{~m}$ and about $0.49 \mathrm{~kg}$, respectively. The robot is connected to a valve unit controlled by a control unit that consists of an embedded controller (Renesas electronics Co. Ltd. SH7125) and transistors. 


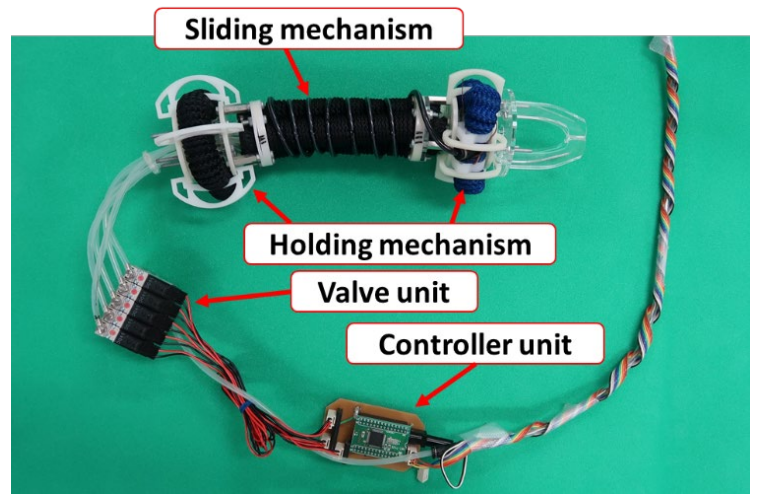

Figure 1. Previous pipe inspection robot using EFPAs.

Figure 2 shows the pipe holding mechanism using the EFPA. The left and right sides of Figure 2 show the view of the tested mechanism when 0 and $500 \mathrm{kPa}$ of supply pressure is applied, respectively. The mechanism consists of a ringshaped EFPA where both ends are connected to a tee joint. Figure 3 shows the construction of the sliding/bending mechanism using EFPAs. In the mechanism, three EFPAs are set in parallel between both disks in every $120^{\circ}$ and are set with a radius of $12 \mathrm{~mm}$ from the central axis of the robot.
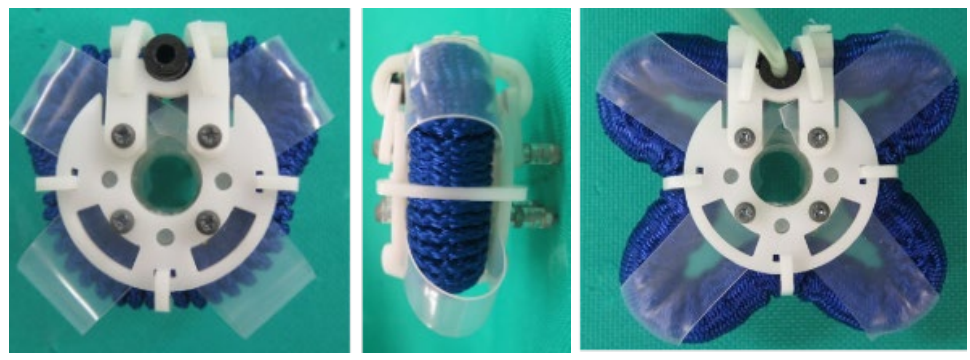

Figure 2. Holding mechanism using EFPA.

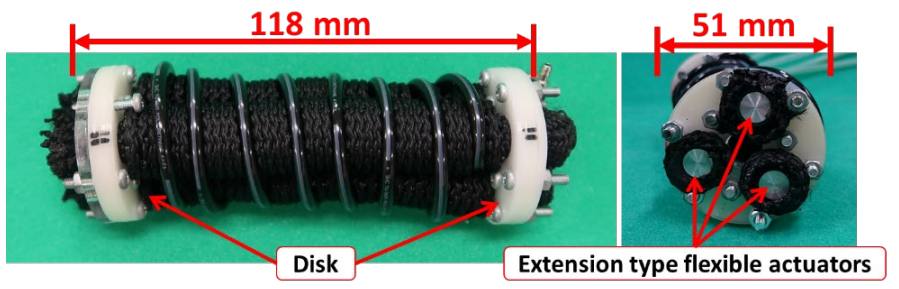

Figure 3. Sliding/bending mechanism using three FFPAs.

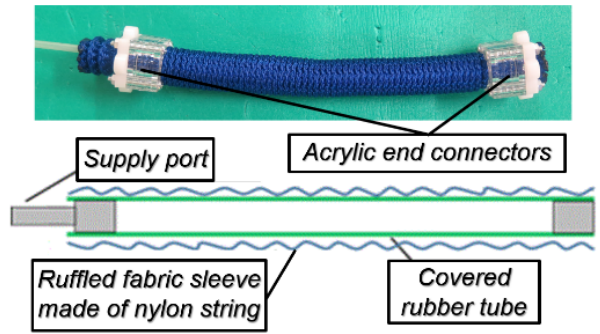

Figure 4. View and inner construction of EFPA.

Figure 4 shows the view and the inner construction of the EFPA. The EFPA consists of a silicone rubber tube (with an inner diameter of $8 \mathrm{~mm}$ and an outer diameter of $10 \mathrm{~mm}$ ) covered with a bellows-shaped ruffled fabric sleeve made of nylon strings and two acrylic end connectors with a supply port. The operating principle of the actuator is as follows; when the supply pressure is applied, the inner rubber tube expands toward both radial and longitudinal directions. As the bellows-shaped ruffled fabric sleeve prevents to expand towards a radial direction, the actuator extends longitudinally. The actuator can extend more than about 2.5 times from its original length. This high extension ratio of $250 \%$ is one of the strongest points of EFPAs.

Figure 5 shows the operating principle of the tested robot. The operating principle of the robot is as follows. First, the pipe holding mechanism at the end of the robot expands so that it can hold the pipe (1). Next, three EFPAs in the sliding mechanism are pressurised; then, the sliding/bending mechanism extends (2). When EFPAs reaches its maximum length, the top of the pipe holding mechanism expands to hold the pipe (3). Then, the pipe holding mechanism at another end of robot contracts (4). Finally, the compressed air in the chambers of three EFPAs is exhausted. At the same time, the 
sliding/bending mechanism contracts and end holding mechanism moves forward (5). By repeating these operations from (1) to (5), the robot can move forward like an inchworm. On the other hand, the backward motion can be realised by using the opposite operation mentioned above. In the case to steer the robot toward the desired direction in pipe joint, first, bending motion of the sliding mechanism is generated by pressurising one or two EFPAs in the mechanism. Next, from this condition of bending motion, three EFPAs in the sliding/bending mechanism are pressurised. Then, the robot can move forward while changing the moving direction. The robot can select six directions by pressurised one or two EFPAs in the sliding/bending mechanism.

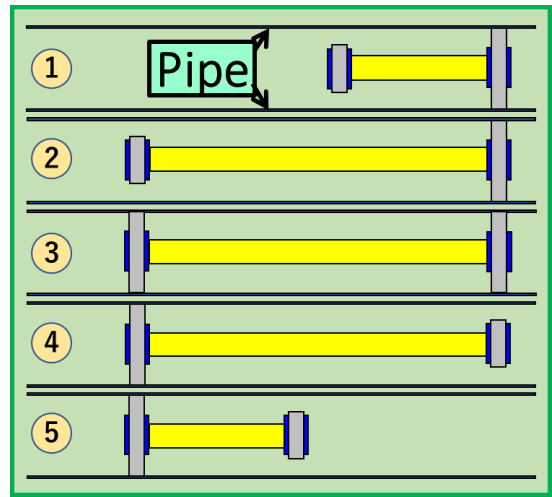

Figure 5. Operating principle of the tested robot.

Figure 6 shows the transient view of the movement of the previous robot when the robot travels in a complex pipe with an inner diameter of $100 \mathrm{~mm}$. In the experiment, three straight pipes with a length of $1 \mathrm{~m}$, an elbow joint and a tee joint were used. The first straight pipe is set on a horizontal plane, and it connects to the second straight pipe with an inclined angle of about $25^{\circ}$ from the horizontal plane through the tee joint. The third straight pipe is set with an inclined angle of about $75^{\circ}$, and it is connected to the second pipe through the elbow joint. The operating procedure of the robot is as follows. First, the robot passes through the straight pipe. Before the robot reaches at elbow or tee joint, the robot bends toward the desired direction. From this condition, three EFPAs in the sliding/bending mechanism are pressurised. Then, the robot can move forward while deforming the shape of its body according to the shape of the inner pipe naturally because of its flexibility. As a result, the robot can pass through joints toward desired passages without stuck. After that, the robot climbs the straight pipe with an inclined angle of $25^{\circ}$ It also turns the elbow joint. Also, it climbs the pipe with an inclined angle of $75^{\circ}$ From the experiment; it could be confirmed that the robot could pass through a complex pipe with a total length of $3 \mathrm{~m}$ within 4 minutes. The average moving speed is $14 \mathrm{~mm} / \mathrm{s}$.

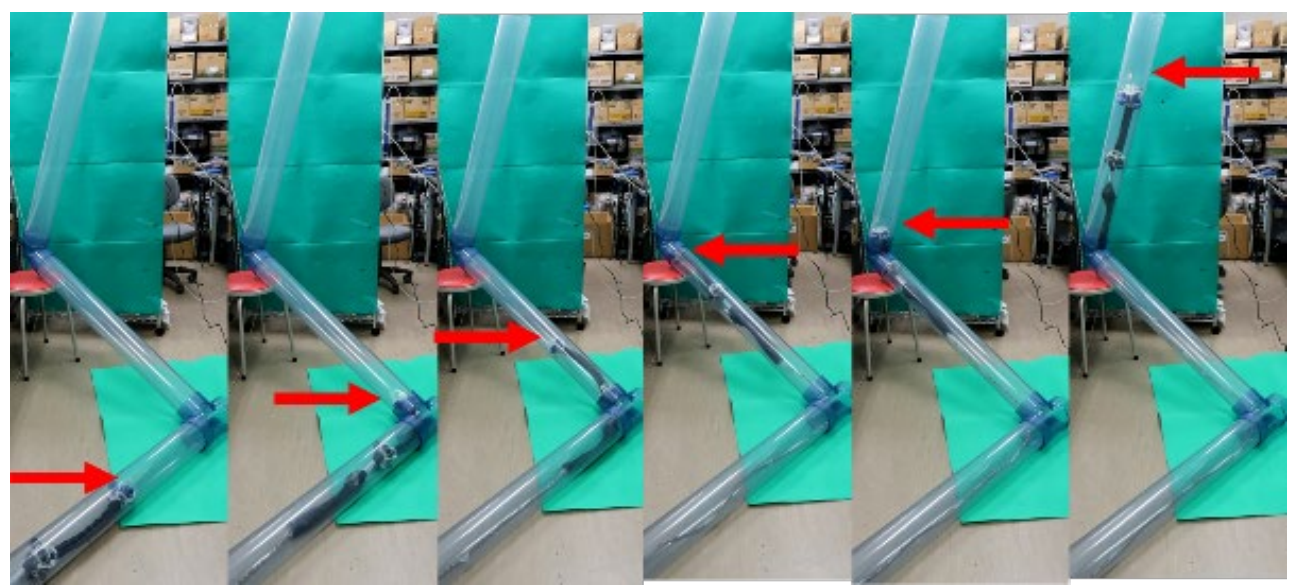

Figure 6. Transient view of the movement of the previous robot in a case when the robot travels in a complex pipe.

\section{DESIGN AND ANALYTICAL MODEL OF SIMPLIFIED PROPULSION MECHANISM}

\section{Design Concept for Simplification}

As mentioned above, in the operation of a pipe inspection robot, one complete cycle of operation to make it move forward is a bit longer. This is due to the sequential operational time of two holding mechanisms and the sliding motion of sliding/bending mechanism primarily to contract the holding mechanism and sliding/ bending mechanism which require longer time compared with the extension. In addition, it seems that it is difficult to reduce the outer diameter of the holding mechanism in order to get the surly holding force. Therefore, rigid parts of the holding mechanism become larger in diameter. It is caused that the holding mechanism can be easily caught in corners compared with the case using the sliding/bending mechanism. In order to reduce the time required for the robot's propulsion operation, it is necessary 
to reduce the number of mechanisms operated by the valve. It is also necessary to reduce the size of the rigid part of the holding mechanism. Therefore, the best solution is to eliminate holding mechanisms and consider a novel propulsion mechanism, that is the simplification of the propulsion mechanism. As a novel method for propulsion, using the difference of frictional force moving forward and backward of the mechanism will be further described.

\section{Analytical Model of Novel Sliding Mechanism}

In order to estimate the propulsion method utilising the difference of frictional force moving forward and backwards, a simple analytical model is proposed, as shown in Figure 7. In the model, the mechanism has two concentrated mass such as end connectors. Between both masses, there is the EFPA to drive them. It is possible to deal with the EFPA as a typical pneumatic cylinder with a damper and a spring. From the equation of motion, the following equation about the motion of front and rear mass are given below.

$$
\begin{aligned}
& m_{0} \cdot \ddot{x_{1}}=F-c \cdot \dot{x_{1}}-k\left(x_{1}-x_{2}\right)-D_{f 1} \\
& m_{0} \cdot \ddot{x_{2}}=F-c \cdot \dot{x_{2}}-k\left(x_{2}-x_{1}\right)-D_{f 2}
\end{aligned}
$$

where, $m_{0}, F, c, k$ and $D_{f}$ represent the concentrated mass of both end connector, generated force of the EFPA, damping coefficient, spring constant of the rubber tube in EFPA and frictional force, respectively. $x_{1}$ and $x_{2}$ are displacement of front and rear mass, respectively. Each mass has a different frictional force of moving forward and backward.

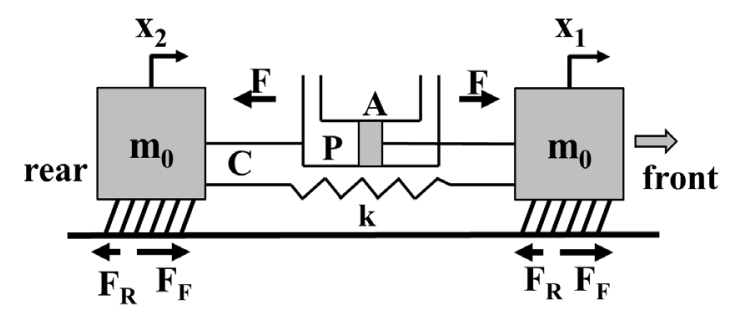

Figure 7. Analytical model of a sliding mechanism.

Figure 8 shows the model of the frictional force that describes the relationship between moving speed and frictional force. In the model, the static friction $F_{S}$ and Coulomb friction $F_{F}$ and $F_{R}$ are changed by the threshold speed $\varepsilon_{v}$ are given by following relations.

$$
\begin{gathered}
|\dot{x}| \leq \varepsilon_{v} \\
D_{f}= \begin{cases}F \cdot \operatorname{sgn}(\dot{x}) & \left(|F| \leq F_{S}\right) \\
F_{s} \cdot \operatorname{sgn}(\dot{x}) & \left(|F|>F_{S}\right)\end{cases} \\
D_{f}= \begin{cases}F_{R} & \left(\dot{x} \mid>\varepsilon_{v} \geq 0\right) \\
-F_{F} & (\dot{x}<0)\end{cases}
\end{gathered}
$$

where, $F_{F}$ and $F_{R}$ mean Coulomb friction toward forward and backward, respectively. Coulomb friction toward forward $\left(F_{F}\right)$ and backward $\left(F_{R}\right)$ are given as follows.

$$
\begin{aligned}
& F_{R}=\mu_{R} \cdot m_{0} \cdot g \\
& F_{F}=\mu_{F} \cdot m_{0} \cdot g
\end{aligned}
$$

where, $\mu_{F}, \mu_{R}$ and $g$ mean the coefficient of friction toward forward and backward and gravity, respectively. The generated force $F$ is given by the equation below.

$$
F=A\left(P-P_{\min }\right)
$$

where, $A, P$ and $P_{\min }$ mean the sectional area of EFPA, input pressure and minimum driving pressure of EFPA, respectively. The pressure change $P$ in the chamber of the EFPA is approximated as a first-order lag element and is given as follows.

$$
T_{P} \cdot \dot{P}+P=a \cdot u
$$

where, $T_{P}, a$ and $u$ mean the time constant of pressure change, the voltage-pressure conversion coefficient of the on/off valve and the input voltage to the valve, respectively. By using these equations, the dynamic behaviour of the sliding mechanism can be calculated. 


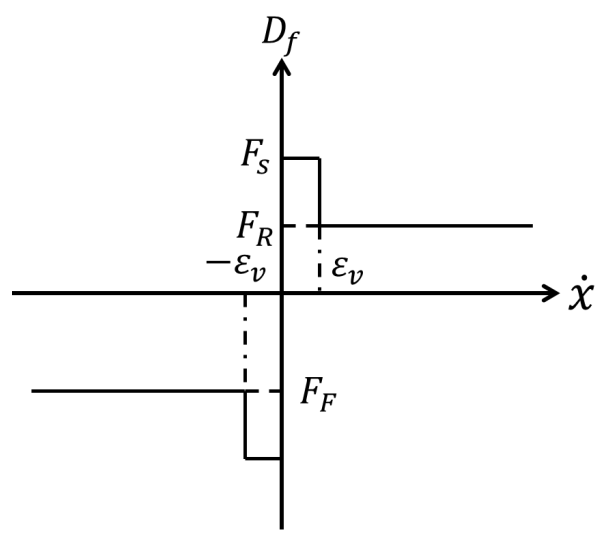

Figure 8. Friction model of the sliding mechanism.

\section{Calculated Results}

Table 1 shows the system parameters value of the sliding mechanism used in the simulation. The coefficient of friction is changed as a variable. In Figure 9, the calculated transient response of the rear displacement $x_{2}$ of the mechanism by using the model mentioned above and system parameters in simulation is shown. In the calculation, the coefficient of friction moving forward is set at 1.0 while the coefficient of backward friction is changed at 1.0, 2.0 and 3.0, respectively. Each line shows rear displacement result using various forward and backward friction ratios that is obtained by using MATLB-Simulink. As a result, the greater the friction ratio of moving forward and backward, the faster the calculated speed.

Table 1. System parameters of the mechanism.

\begin{tabular}{lc}
\hline Parameter & Value \\
\hline$A\left(\mathrm{~m}^{2}\right)$ & 0.00785 \\
$a(\mathrm{kPa} / \mathrm{v})$ & 100 \\
$c(\mathrm{Ns} / \mathrm{m})$ & 3 \\
$F_{S}(\mathrm{~N})$ & 6 \\
$k(\mathrm{~N} / \mathrm{m})$ & 200 \\
$m_{0}(\mathrm{~kg})$ & 0.2 \\
$P_{\min }(\mathrm{kPa})$ & 100 \\
$T_{p}(\mathrm{~s})$ & 0.06 \\
$\mu_{F}$ & $1 \sim 7$ \\
$\mu_{R}$ & 1 \\
\hline
\end{tabular}

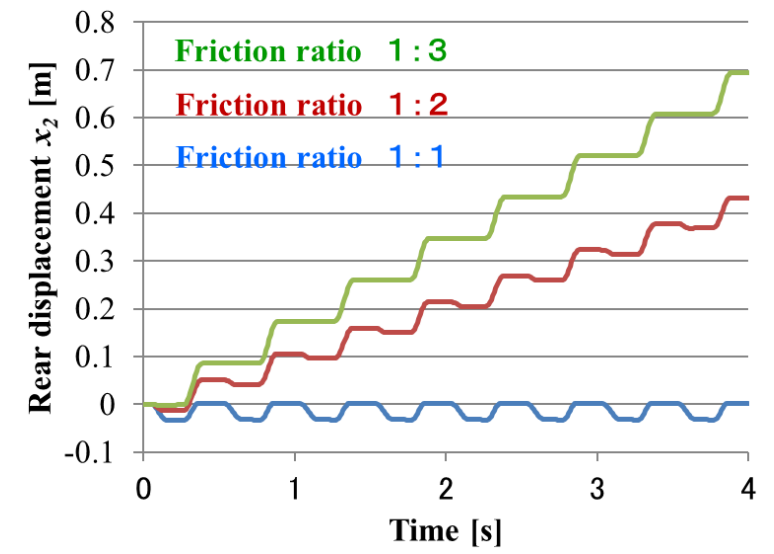

Figure 9. Calculated transient response of the rear displacement of the sliding mechanism using a various ratio of coefficient of friction moving forward and backward. 


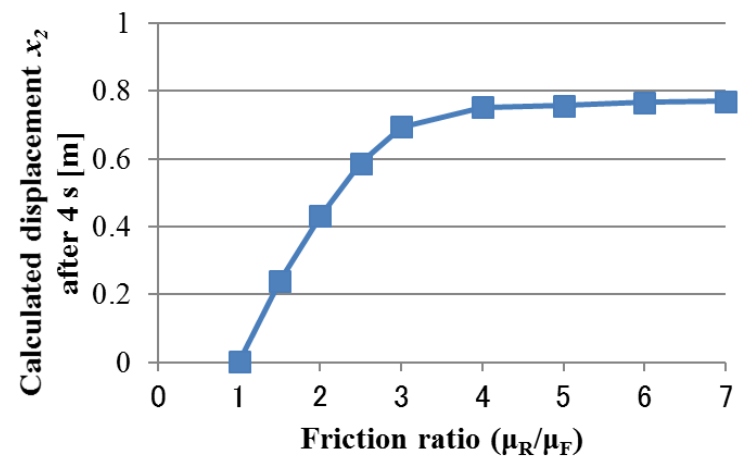

Figure 10. Relation between the friction ratio of moving forward and backward and calculated displacement of the mechanism after $4 \mathrm{~s}$.

Figure 10 shows the relationship between the friction ratio of moving forward and backward and the calculated displacement $x_{2}$ of the mechanism after 4 seconds from the beginning of the calculation. From Figure 10, the calculated displacement increases in line with the increase of friction ratio until it reaches saturated condition at friction ratio more than 4. Consequently, in designing the mechanism, the friction ratio of moving forward and backward is set to 4 .

\section{WRIGGLING TYPE SLIDING/BENDING MECHANISM}

\section{Construction}

In order to get different friction ratio of moving forward and backward, the sliding mechanism whose end connector can push forward the pipe while wriggling its body is proposed and tested. Figure 11 shows the view of the tested wriggling type sliding/bending mechanism. The mechanism consists of the sliding/bending mechanism, as shown in Figure 3 and front and rear connectors. Compared with the previous sliding/bending mechanism, parallel arranged EFPAs are not covered by the coil type tube. As a front connector, the pointed acrylic cover is used to reduce the friction. As a rear connector, the disk covered by rubber tube. The disk is also connected to three metal rods whose ends are covered by silicon rubber tubes. The diameter of rear disk is set at $41 \mathrm{~mm}$ so that it can not touch the pipe while the mechanism with an outer diameter of $44 \mathrm{~mm}$ is keeping straight. The total length and mass of the mechanism is about $220 \mathrm{~mm}$ and $180 \mathrm{~g}$, respectively.

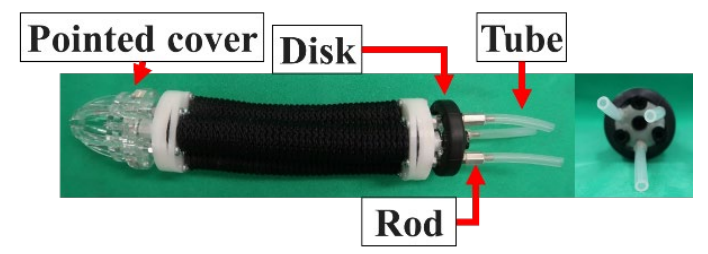

Figure 11. Construction of wriggling type sliding/bending mechanism.

\section{Operating Principle}

Figure 12 shows the operating principle of the wriggling type sliding/bending mechanism. The operating principle of the mechanism is as follows. Firstly, when one EFPA in the mechanism is pressurised, then the mechanism bends. Simultaneously, both front and rear connectors contact with the surface of the pipe and press the pipe, as shown in Figure 12(2). In the condition, rest two EFPAs are pressurised, that is, all EFPAs are pressurised. Then, while the rear connector is pushing the inner wall of the pipe, the mechanism extends forward, as shown in (2)-(3). After finishing to extend all EFPAs as shown in (4), by exhausting inner pressure of three EFPAs one by one according to the time chart of valve operation as shown in Figure 13, the mechanism wriggles and move forward as follows. Each valve is connected to each EFPA in the mechanism. After all the EFPAs contact with the wall of the pipe one by one, the mechanism changes the position forward using the force that generated by friction difference between the front and rear connectors as shown in (5). Finally, since the centre of gravity of the robot is moving forward, it will move forward slightly when it is contracted (6). 


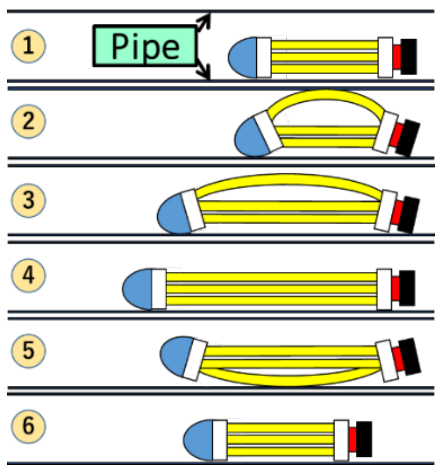

Figure 12. Operating principle of wriggling type sliding/bending mechanism.

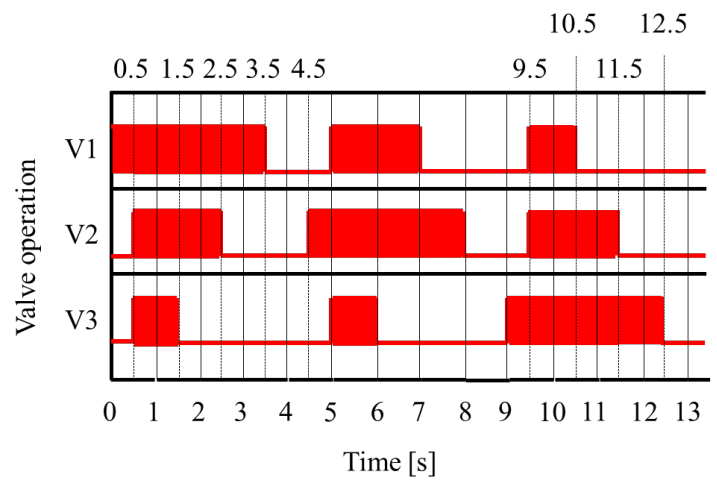

Figure 13. Time chart of operation of each valve that connects to each EFPA for moving forward.

(The red marker indicates that the valve opens and supplies compressed air to the EFPAs. The blank means that valve closes and works as an exhaust.).

\section{Experimental Results}

Figure 14 shows the schematic diagram of a control system to drive the sliding/bending mechanism. The system consists of the tested mechanism, three on/off control valves (Koganei Co. Ltd., G010HE1) and an embedded controller (Renesas Electronics, SH7125). Each valve is operated through a transistor and I/O port in the embedded controller according to the time chart of valve operation. The control procedure is described as follows. The operator sends digital code through a serial communication cable using a personal computer. The embedded controller selects to drive a suitable valve according to the digital code. Specifically, the embedded controller selects the to the preinstalled sequential program according to the digital code. Each valve is driven based on the sequential program that is programmed the timing of valve operation for such as moving forward and backward. The timing of valve operation is adjusted by using timer function in the embedded controller.

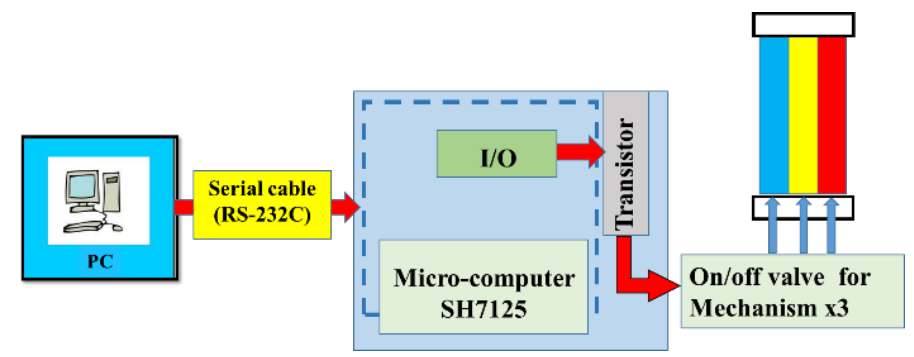

Figure 14. Schematic diagram of control system to drive the mechanism.

Figure 15 shows the transient view of the movement of the tested wriggling type mechanism. In the experiment, three EFPAs are driven according to the time chart, as shown in Figure 13. Referring to Figure 13, the red marker indicates that the valve opens and supplies compressed air to the EFPAs. The blank means that the valve closes and works as an exhaust. The time chart is determined by trial and error so that the mechanism can move forward in the pipe. From Figure 15 , it can be seen that the mechanism can move forward by pushing the pipe using the end connector while wriggling its body. As a result, it can be confirmed that the mechanism can move forward with an average speed of $6 \mathrm{~mm} / \mathrm{s}$. The speed was measured by moving speed from the video. 


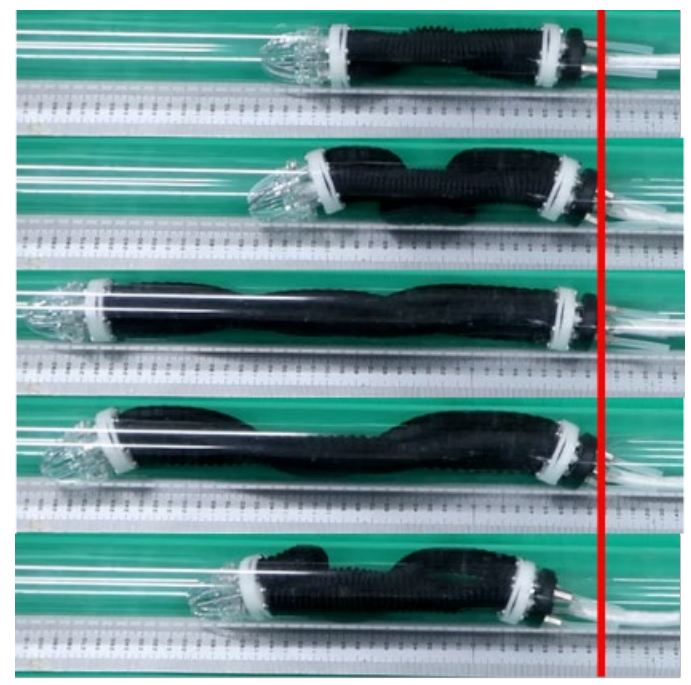

Figure 15. Transient view of movement of wriggling type mechanism.

\section{CILIA TYPE SLIDING/BENDING MECHANISM}

\section{Construction and Operating Principle}

In the previous mechanism, as shown in Figure 3, the mechanism can generate moving force by wriggling its body. However, the mechanism requires the time for wriggling motion. Instead of its motion, by using cilia that has naturally difference of friction for moving forward and backward, the mechanism can be simplified. Based on this concept, the cilia type sliding/bending mechanism is proposed and tested. Figure 16 shows the construction of the cilia type sliding/bending mechanism. The mechanism consists of the typical sliding/bending mechanism using parallel arranged three EFPAs and 15 cilia plates. The cilia plate is made of multilayered nylon with a thickness of $0.4 \mathrm{~mm}$. The shape of the plate is shown in Figure 16(b). Each plate has three holes with a diameter of $17.5 \mathrm{~mm}$, and it fitted to bellows on EFPA. These cilia plates also work to keep a parallel arrangement of three EFPAs. The difference of frictional force can be realised by giving the inclined angle to these cilia plates setting on EFPA against to the longitudinal axis of the mechanism. The total length of the mechanism is about $130 \mathrm{~mm}$, and the mass of the mechanism becomes lighter compared with the wriggling mechanism, as shown in Figure 11, that is about $120 \mathrm{~g}$. The operating principle of this mechanism is simpler compared with the previous mechanism. By repeating to pressurise and exhaust three EFPAs at the same time, the cilia type mechanism is able to move forward by only using the different friction of cilia.

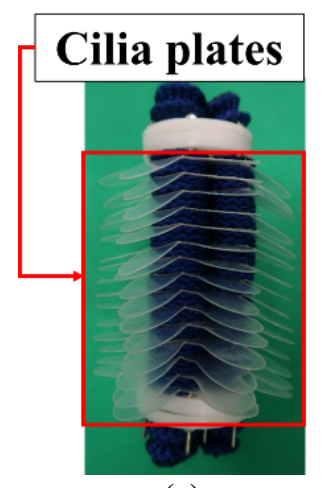

(a)

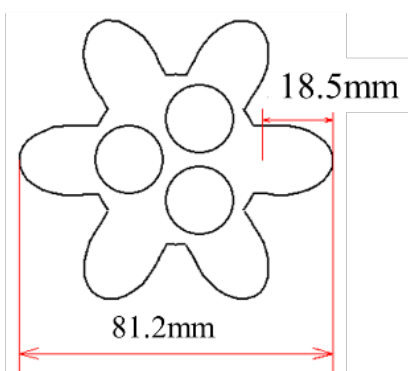

(b)

Figure 16. Construction of cilia type sliding/bending mechanism.

\section{Experimental Results}

Figure 17 shows the transient view of the movement of the cilia type sliding/bending mechanism. In the driving test, supply and exhaust in chambers of EFPAs were alternatively changed every 2 seconds. It can be seen that the displacement of the mechanism for one cycle motion is shorter than the case using the previous one. However, as one cycle of the tested mechanism is shorter than the previous one, the almost same moving speed of $5 \mathrm{~mm} / \mathrm{s}$ can be obtained. The speed was also measured by moving speed from the video. 


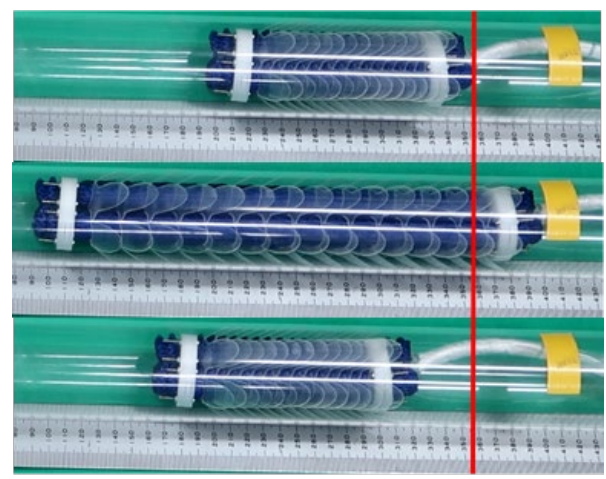

Figure 17. Transient view of movement of cilia type sliding/bending mechanism.

Figure 18 shows the transient view of the movement of the tested mechanism in the pipe that includes water. In the experiment, a hemispherical plastic cap with a diameter of $49 \mathrm{~mm}$ is used as a front connector to reduce the resistance of water. Water is $60 \%$ filled in a pipe with an inner diameter of $56 \mathrm{~mm}$. From Figure 18, it can be seen that the displacement of the mechanism for one cycle motion a slightly decreased compared using a dry pipe. This is because the friction ratio turns out to be smaller by filling the water. The moving speed of $3.6 \mathrm{~mm} / \mathrm{s}$ can be obtained. As a result, it can be confirmed that the tested mechanism can move forward in the pipe containing $60 \%$ of water. It can also be observed that the tested mechanism can work well in a wet pipe.

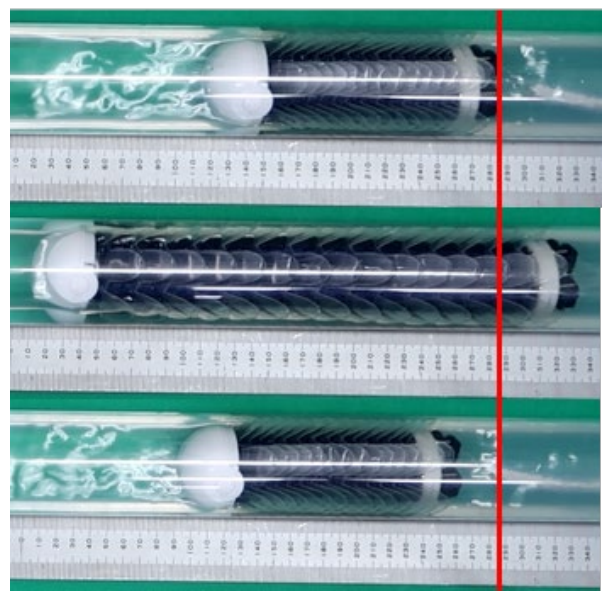

Figure 18. Result of driving test in case using pipe that water is $60 \%$ filled.

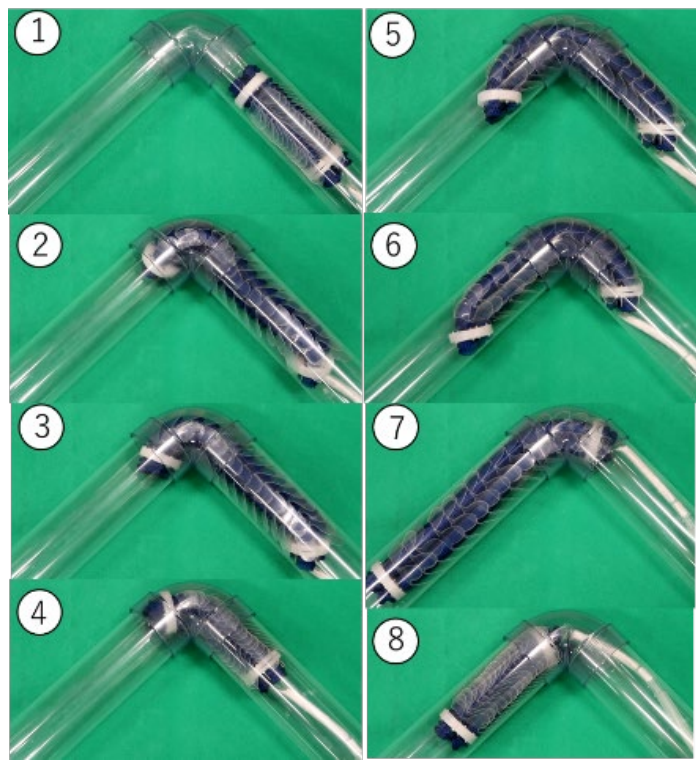

Figure 19. Result of driving test in a case when the mechanism passes through the corner.

Figure 19 shows the result of a driving test when the mechanism passes through the corner. In the first trial of the experiment, the typical motion for moving forward that all EFPAs were driven at the same time was given. However, the mechanism could not pass through the corner. Therefore, in the experiment, the bending motion was given for the mechanism just before travelling the corner. After sticking the front connector into the corner, the typical motion for 
moving forward was given to the mechanism. By this method, the mechanism could pass through the corner. As more interesting, it can be found that the mechanism could naturally twist toward suitable bending direction even if the bending motion toward the opposite direction was applied to the mechanism as shown in Figure 19.

\section{CONCLUSION}

The simplified pipe inspection robot that can travel in a damp and wet pipe is summarised as follows:

i. To estimate the performance of the propulsion mechanism in pipe utilising the difference of frictional force moving forward and backward theoretically, the analytical model of the sliding mechanism using extension type flexible pneumatic actuators ("EFPAs" for short) was proposed. The simulation using various combinations of friction between moving forward and backward was carried out by using MATLAB. As a result, it can be confirmed that the speed of the mechanism is saturated when the friction ratio is set at four and it is better to set as the desired value to design the mechanism.

ii. As a propulsion mechanism using friction difference of moving forward and backward, the "wriggling type" sliding/bending mechanism is proposed and tested. The mechanism consists of parallel arranged three EFPAs, a front connector with low sliding friction and a rear connector with larger sliding friction. The timing chart of valve operation for three EFPAs in the mechanism for propulsion could be obtained by trial and error. As a result, it can be confirmed experimentally that the mechanism can move forward by pushing the pipe using a rear connector while wriggling its body. It also found that the mechanism can move forward with a speed of $6 \mathrm{~mm} / \mathrm{s}$.

iii. To simplify the sliding propulsion mechanism, the "cilia type" sliding/bending mechanism using cilia that naturally has the difference of friction for moving forward and backward was proposed and tested. As a result of driving test, it can be confirmed that displacement of the mechanism for one cycle is shorter than the case using "wriggling type" one. However, as one cycle is shorter than the "wriggling type", almost the same moving speed of $5 \mathrm{~mm} / \mathrm{s}$ can be obtained. It can also be confirmed that the tested "cilia type" mechanism can move forward in the pipe that contains $60 \%$ of water with a speed of $3.6 \mathrm{~mm} / \mathrm{s}$. Interestingly, it can be found that the mechanism could naturally twist toward suitable bending direction even if the bending motion toward the opposite direction is applied to the mechanism.

\section{ACKNOWLEDGEMENT}

This research was supported by the MEXT in Japan through a Financial Assistance Program for QOL Innovative Research (2012-2016) and a Grant-in-Aid for Scientific Research (C) (Subject 16K06202 \& 19K04265).

\section{REFERENCES}

[1] Current situation of water supply in japan (in Japanese). Retrieved from: https://www.mhlw.go.jp/ seisakunitsuite/bunya/topics/bukyoku/kenkou/suido/newvision/chiikikondan/10/suishin_kondan_10-1.pdf.

[2] Yuan J, Wu X, Kang Y, Huang C. Development of an inspection robot for long distance transmission pipeline on-site overhaul. International Journal of Industrial Robot, 2009; 36: 546-550.

[3] Dertien E, Stramigioli S, Pulles K. Development of an inspection robot for small diameter gas distribution mains. In: 2011 IEEE International Conference on Robotics and Automation, Shanghai, China, pp.281-294; 2011

[4] Nagatani K, Kuze M, Yoshida K. Development of a transformable mobile robot with a variable wheel diameter. Journal of Robotics and Mechatronics, 2007; 19(3): 252-257.

[5] Kawasaki T, Akagi T, Dohta S, Harihara K. Development of a flexible sliding mechanism using flexible pneumatic cylinder for rescue robot. In: 11th International Conference on Fluid $11^{\text {th }}$ International Conference on Fluid Control, Measurements, and Visualization, Keelung, Taiwan, 41.pdf, pp.1-6; 2011.

[6] Akagi T, Dohta S, Wang L, Kawasaki T. Development of sliding type inspection robot using flexible pneumatic cylinder. International Journal of Advanced Mechatronic Systems, 2013; 5: 87-94.

[7] Akagi T, Dohta S, Qiu H. Pipe inspection robot using flexible pneumatic cylinder built-in pneumatic driving system. In: International Forum on Systems and Mechatronics, Tainan, Taiwan, pp. 1-6; 2014.

[8] Choi K, Dohta S, Akagi T, Ninomiya S. Development of pipe holding mechanism for pipe inspection robot using flexible pneumatic cylinder. In: 2016 International Conference on Mechanical, Manufacturing, Modeling and Mechatronics, Kuala Lumpur, Malaysia, pp. 529-536; 2016.

[9] Kusunose K, Akagi T, Dohta S, Kobayashi W, Nakagawa K. Development of pipe holding mechanism and bending unit using extension type flexible actuator for flexible pipe inspection robot. International Journal of Mechanical Engineering and Robotics Research 2019; 8(1): 129-134.

[10] Shinohara T, Kusunose K, Akagi T, Dohta S et al. Improvement of pipe inspection robot using extension type flexible pneumatic actuators. International Journal of Mechanical Engineering and Robotics Research 2020; 9(6): 894-899. 